\title{
The Impact of Fixed and Moving Scatterers on the Statistics of MIMO Vehicle-to-Vehicle Channels
}

\author{
Ali Chelli and Matthias Pätzold \\ University of Agder \\ Norway
}

\section{Introduction}

In most countries, the reduction in road casualties is a top priority. The intelligent transportation system (ITS) is a national program in the U.S. aiming to improve road safety. In order to deploy the ITS, vehicle-to-vehicle (V2V) communication techniques are needed. The dedicated short range communication (DSRC) standard (ASTM, 2003) is designed for V2V communications. Several task groups are working on this standard including the IEEE 802.11p (802.11p, 2006) and the IEEE 1609.4 (WAVE, 2005).

The statistical properties of $\mathrm{V} 2 \mathrm{~V}$ channels are different from the conventional fixed-to-mobile channels. Therefore, new channel models are needed for V2V communications. The geometrical two-ring model (Patel et al., 2003), (Pätzold et al., 2005) has been proposed for V2V communications. Unfortunately, this channel model cannot be used to describe propagation conditions along streets for V2V channels. In fact, in such an environment, the wave-guiding along the street has a dominant effect. It was suggested in (Molisch et al., 1999) that the wave-guiding can be implemented by using geometry-based channel models, where the scatterers are located on straight lines. The geometrical street model introduced in (Chelli \& Pätzold, 2007) captures the propagation effects if the communicating vehicles are moving along a straight street. The street model has been extended with respect to multiple clusters of scatterers as well as to frequency selectivity in (Chelli \& Pätzold, 2008). In (Zajić et al., 2008), a $3 \mathrm{D}$ channel model for V2V communications has been proposed. Measurement results in (Zajić et al., 2008) have shown that for vehicles driving in the middle lanes of highways or in urban environment, the double-bounce rays caused by fixed scatterers are dominant. In contrast to our model presented in (Chelli \& Pätzold, 2007) and (Chelli \& Pätzold, 2008) where single-bounce scattering is assumed, we assume double-bounce scattering for fixed scatterers. Double-bounce models are fundamentally different from single-bounce models. In fact, for double-bounce models the angles of departure $(\mathrm{AoD})$ and the angles of arrival $(\mathrm{AoA})$ are independent. This is in contrast to single-bounce models where the AoD and the AoA are closely related. Due to this dissimilarity, the statistical properties of double-bounce models and single-bounce models are different. Therefore, double-bounce models should be studied carefully.

Furthermore, the presence of moving scatterers in a highway environment has a big impact on the channel behaviour. For this reason, we study the effect of passing vehicles on the channel statistical properties. Measurement results in (Millott, 1994) have shown that the amplitude of waves scattered from more than one vehicle is small and the practical impact of vehicular 
scattering is confined to single-bounce rays. Therefore, double-bounce scattering from moving scatterers has been neglected in our model. When scatterers are moving with a high speed relatively to the transmitter (receiver) the $\mathrm{AoD}(\mathrm{AoA})$ becomes time-variant resulting in a nonstationary channel model. However, when vehicles are facing road congestion, the relative speed of the cars in the vicinity of the transmitter or the receiver is low. In such conditions, we can still consider the AoD and the AoA as non-time-variant during a sufficiently large period of time. This assumption can be accepted especially if the scatterers are moving in the same direction as the transmitter and the receiver.

The remainder of the chapter is organized as follows. In Section II, the geometrical street model is presented. Based on this geometrical model, we derive a reference model in Section III. In Section IV, we study the correlation properties of the proposed channel model. Numerical results of the correlation functions are presented in Section $\mathrm{V}$ to validate all theoretical results by simulations. Finally, Section VI provides some concluding remarks.

\section{The Geometrical Street Model}

A typical highway propagation environment for $\mathrm{V} 2 \mathrm{~V}$ communication is presented in Fig. 1. The highway encompasses three lanes used for traffic in the same direction. We can distinguish between two types of scatterers namely fixed scatterers and moving scatterers. The fixed scatterers are represented by the buildings located on both sides of the street, while the moving scatterers are the vehicles in the vicinity of the transmitter $\mathrm{MS}_{T}$ and the receiver $\mathrm{MS}_{R}$. In order to be able to develop an appropriate channel model for the propagation scenario presented in Fig. 1, we first need to produce a representative geometrical model for such an environment. Towards this aim, we model each building by a cluster of scatterers located on a straight line on the left or right hand side of the street. A vehicle can be modeled by a cluster of scatterers located on a line as well. The fixed clusters are represented by solid lines while the moving clusters are represented by dashed lines. The geometrical street model encompassing fixed and moving scatterers is illustrated in Fig. 2. The fixed scatterers around the transmitter (receiver) are denoted by $S_{m}^{T}\left(S_{n}^{R}\right)$. The moving scatterers are designated by $S_{p}^{M}$. The propagation environment encompasses $\mathcal{C}^{T}\left(\mathcal{C}^{R}\right)$ fixed clusters around the transmitter (receiver) and $\mathcal{C}^{M}$ moving clusters. For fixed clusters, the AoD is referred to as $\alpha_{m}^{T}$, whereas the AoA is denoted by $\beta_{n}^{R}$. For moving clusters, the symbols $\alpha_{p}^{M}$ and $\beta_{p}^{M}$ stand for the AoD and the AoA, respectively. It has to be noted that for fixed clusters the AoD and the AoA are independent since double-bounce scattering is assumed. For moving clusters, the $\mathrm{AoD}$ and the AoA are closely related due to the single-bounce scattering assumption. All scatterers belonging to a given moving cluster have the same velocity $\mathrm{v}_{S}$ and the same direction of motion $\phi^{S}$. The transmitter and the receiver are moving with velocity $\mathrm{v}_{T}$ and $\mathrm{v}_{R}$, respectively. The angle of motion of the transmitter and the receiver w.r.t the $x$-axis is referred to as $\phi^{T}$ and $\phi^{R}$, respectively. Moreover, the transmitter (receiver) is equipped with $M_{T}\left(M_{R}\right)$ antenna elements. The antenna element spacing at the transmitter and the receiver antenna are denoted by $\delta_{T}$ and $\delta_{R}$, respectively. The angle $\gamma_{T}\left(\gamma_{R}\right)$ describes the tilt angle of the transmit (receive) antenna array.

\section{The Reference Model}

Starting from the geometrical model shown in Fig. 2, we derive a reference model for the MIMO V2V channel. First, we consider the case where we have one moving cluster and two fixed clusters: one cluster is near to the transmitter and the other cluster is close to the 


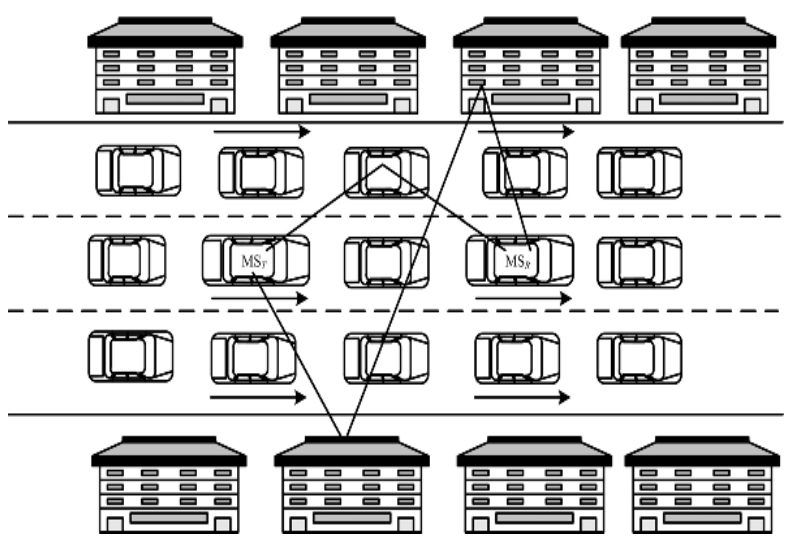

Fig. 1. A highway propagation environment for vehicle-to-vehicle communications under congestion conditions.

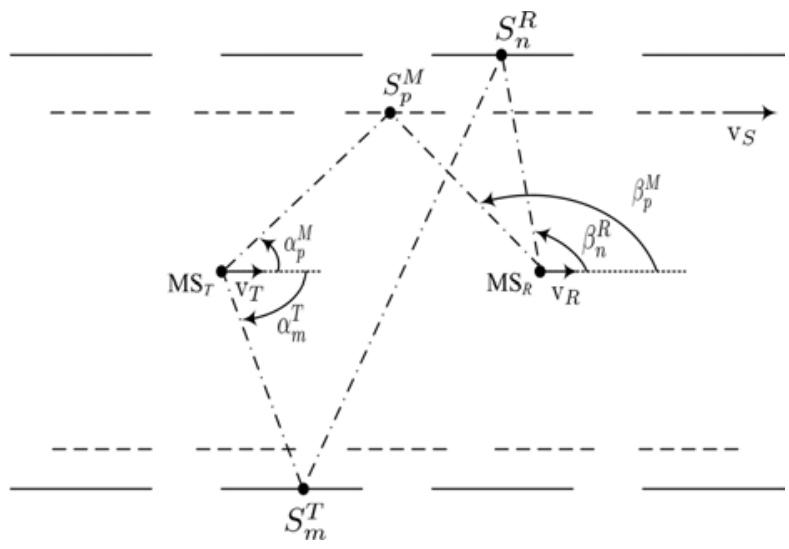

Fig. 2. The geometrical street model encompassing moving and fixed scatterers.

receiver. The total number of fixed scatterers around the transmitter (receiver) is denoted by $M(N)$, while the number of moving scatterers is referred to as $P$. The complex channel gain $g_{k l}(t)$ describing the link between the $l$ th transmit antenna element $A_{l}^{T}\left(l=1,2, \ldots, M_{T}\right)$ and the $k$ th receive antenna element $A_{k}^{R}\left(k=1,2, \ldots, M_{R}\right)$ of the underlying $M_{T} \times M_{R}$ MIMO V2V channel model can be expressed as $g_{k l}(t)=g_{k l}^{\mathrm{F}}(t)+g_{k l}^{\mathrm{M}}(t)$. The term $g_{k l}^{\mathrm{F}}(t)$ stands for the channel gain due to double-scattering from the fixed clusters. The channel gain caused by the moving cluster is denoted by $g_{k l}^{\mathrm{M}}(t)$. We assume that the line-of-sight component is obstructed. Next, we derive analytical expressions of the channel gains $g_{k l}^{\mathrm{F}}(t)$ and $g_{k l}^{\mathrm{M}}(t)$.

\subsection{The Channel Gain Due to Fixed Scatterers}

The plane wave emitted from the $l$ th transmit antenna element $A_{l}^{T}$ travels over the scatterers $S_{m}^{T}$ and $S_{n}^{R}$ before impinging on the $k$ th receive antenna element $A_{k}^{R}$. Based on the geometrical model in Fig. 2, the channel gain, due to double-scattering from fixed clusters, $g_{k l}^{\mathrm{F}}(t)$ can be 
written as

$$
g_{k l}^{\mathrm{F}}\left(\vec{r}_{T}, \vec{r}_{R}\right)=\sum_{m, n=1}^{M, N} c_{m n} e^{j\left(\theta_{m n}+\vec{k}_{m}^{T} \cdot \vec{r}_{T}-\vec{k}_{n}^{R} \cdot \vec{r}_{R}-k_{0} d_{m n}\right)}
$$

where $c_{m n}$ and $\theta_{m n}$ stand for the joint gain and the joint phase shift resulting from the interaction with the fixed scatterers $S_{m}^{T}$ and $S_{n}^{R}$. The joint channel gain can be written as $c_{m n}=1 / \sqrt{M N}$, while the joint phase shift can be expressed as $\theta_{m n}=\left(\theta_{m}+\theta_{n}\right) \bmod 2 \pi$, where mod stands for the modulo operation. The terms $\theta_{m}$ and $\theta_{n}$ are the phase shifts associated with the scatterers $S_{m}^{T}$ and $S_{n}^{R}$, respectively. It has to be noted that $\theta_{m}, \theta_{n}$, and $\theta_{m, n}$ are independent identically distributed (i.i.d.) random variables uniformly distributed over $[0,2 \pi)$.

The second phase term in (1), $\vec{k}_{m}^{T} \cdot \vec{r}_{T}$, is related to the transmitter movement. The symbol $\vec{k}_{m}^{T}$ denotes the wave vector pointing in the propagation direction of the $m$ th transmitted plane wave, and $\vec{r}_{T}$ is the spatial translation vector of the transmitter. The scalar product $\vec{k}_{m}^{T} \cdot \vec{r}_{T}$ can be expressed as

$$
\vec{k}_{m}^{T} \cdot \vec{r}_{T}=2 \pi f_{\max }^{T} \cos \left(\alpha_{m}^{T}-\phi^{T}\right) t
$$

where $f_{\max }^{T}=\mathrm{v}_{T} / \lambda$ stands for the maximum Doppler frequency associated with the mobility of the transmitter. The symbol $\lambda$ denotes the wavelength.

The third phase term in (1), $\vec{k}_{n}^{R} \cdot \vec{r}_{R}$, is caused by the receiver movement. The symbol $\vec{k}_{n}^{R}$ denotes the wave vector pointing in the propagation direction of the $n$th received plane wave, and $\vec{r}_{R}$ is the spatial translation vector of the receiver. The scalar product $\vec{k}_{n}^{R} \cdot \vec{r}_{R}$ can be written as

$$
\vec{k}_{n}^{R} \cdot \vec{r}_{R}=-2 \pi f_{\max }^{R} \cos \left(\beta_{n}^{R}-\phi^{R}\right) t
$$

where $f_{\max }^{R}=\mathrm{v}_{R} / \lambda$ stands for the maximum Doppler frequency due to the receiver movement.

The term $k_{0} d_{m n}$ in (1) is associated with the total travelled distance and can be expressed as

$$
k_{0} d_{m n}=\frac{2 \pi}{\lambda}\left(D_{l m}+D_{m n}+D_{n k}\right)
$$

where $D_{l m}$ denotes the distance from the $l$ th transmit antenna element $A_{l}^{T}$ to the scatterer $S_{m}^{T}$. The symbol $D_{m n}$ stands for the distance between the scatterers $S_{m}^{T}$ and $S_{n}^{R}$. The term $D_{n k}$ denotes the distance from the scatterer $S_{n}^{R}$ to the $k$ th receive antenna element $A_{k}^{R}$. The distances $D_{l m}$ and $D_{n k}$ can be approximated as

$$
\begin{aligned}
& D_{l m} \approx D_{m}^{T}-\left(M_{T}-2 l+1\right) \frac{\delta_{T}}{2} \cos \left(\alpha_{m}^{T}-\gamma_{T}\right) \\
& D_{n k} \approx D_{n}^{R}-\left(M_{R}-2 k+1\right) \frac{\delta_{R}}{2} \cos \left(\beta_{n}^{R}-\gamma_{R}\right)
\end{aligned}
$$

where $D_{m}^{T}$ denotes the distance from the transmitter to the scatterer $S_{m}^{T}$ and $D_{n}^{R}$ corresponds to the distance from the receiver to the scatterer $S_{n}^{R}$. After substituting (2)-(6) in (1) the channel gain caused by double-scattering from fixed clusters can be expressed as

$$
g_{k l}^{\mathrm{F}}(t)=\sum_{m, n=1}^{M, N} \frac{a_{m}^{T} b_{n}^{R} c_{m n}^{T R}}{\sqrt{M N}} e^{j\left(2 \pi\left(f_{m}^{T}+f_{n}^{R}\right) t+\theta_{m n}\right)}
$$


where

$$
\begin{aligned}
a_{m}^{T} & =e^{j \pi \frac{\delta_{T}}{\lambda}\left(M_{T}-2 l+1\right) \cos \left(\alpha_{m}^{T}-\gamma_{T}\right)} \\
b_{n}^{R} & =e^{j \pi \frac{\delta_{R}}{\lambda}\left(M_{R}-2 k+1\right) \cos \left(\beta_{n}^{R}-\gamma_{R}\right)} \\
c_{m n}^{T R} & =e^{-j \frac{2 \pi}{\lambda}\left(D_{m}^{T}+D_{m n}+D_{n}^{R}\right)} \\
f_{m}^{T} & =f_{\max }^{T} \cos \left(\alpha_{m}^{T}-\phi^{T}\right) \\
f_{n}^{R} & =f_{\max }^{R} \cos \left(\beta_{n}^{R}-\phi^{R}\right) .
\end{aligned}
$$

It has to be mentioned that the envelope $\left|g_{k l}^{\mathrm{F}}(t)\right|$ follows a double Rayleigh distribution since double-bounce scattering is assumed (Salo et al., 2006).

\subsection{The Channel Gain Due to Moving Scatterers}

The plane wave emitted from the $l$ th transmit antenna element $A_{l}^{T}$ travels over the scatterer $S_{p}^{M}$ before impinging on the $k$ th receive antenna element $A_{k}^{R}$. Based on the geometrical model in Fig. 2, the channel gain $g_{k l}^{\mathrm{M}}(t)$ of the moving cluster can be expressed as

$$
g_{k l}^{\mathrm{M}}\left(\vec{r}_{T}, \vec{r}_{R}, \vec{r}_{S}\right)=\sum_{p=1}^{P} c_{p} e^{j\left(\theta_{p}+\vec{k}_{p}^{T} \cdot \vec{r}_{T}-\vec{k}_{p}^{R} \cdot \vec{r}_{R}-\vec{k}_{p}^{T} \cdot \vec{r}_{S}+\vec{k}_{p}^{R} \cdot \vec{r}_{S}-k_{0} d_{p}\right)}
$$

where $c_{p}$ and $\theta_{p}$ represent the gain and the phase shift resulting from the interaction with the moving scatterer $S_{p}^{M}$, respectively. The channel gain is given by $c_{p}=1 / \sqrt{P}$, while the phase shifts $\theta_{p}$ are i.i.d. random variables uniformly distributed over $[0,2 \pi)$. The phase changes $\vec{k}_{p}^{T} \cdot \vec{r}_{T}$ and $\vec{k}_{p}^{R} \cdot \vec{r}_{R}$ are associated with the movement of the receiver and the transmitter, respectively, and can be written as

$$
\begin{aligned}
\vec{k}_{p}^{T} \cdot \vec{r}_{T} & =2 \pi f_{\max }^{T} \cos \left(\alpha_{p}^{M}-\phi^{T}\right) t \\
\vec{k}_{p}^{R} \cdot \vec{r}_{R} & =-2 \pi f_{\max }^{R} \cos \left(\beta_{p}^{M}-\phi^{R}\right) t .
\end{aligned}
$$

The spatial translation $\vec{r}_{S}$ of the moving scatterer $S_{p}^{M}$ influences the wave emitted from the transmitter resulting in a phase change $\vec{k}_{p}^{T} \cdot \vec{r}_{S}$. Moreover, the scatterer $S_{p}^{M}$ interacts with the wave reflected to the receiver resulting in a phase change $\vec{k}_{p}^{R} \cdot \vec{r}_{S}$. These phase changes can be expressed as

$$
\begin{aligned}
& \vec{k}_{p}^{T} \cdot \vec{r}_{S}=2 \pi f_{\max }^{S} \cos \left(\alpha_{p}^{M}-\phi^{S}\right) t \\
& \vec{k}_{p}^{R} \cdot \vec{r}_{S}=-2 \pi f_{\max }^{S} \cos \left(\beta_{p}^{M}-\phi^{S}\right) t
\end{aligned}
$$

where $f_{\max }^{S}=\mathrm{v}_{S} / \lambda$ is referred to as the maximum Doppler frequency caused by the moving cluster. Recall that all scatterers $S_{p}^{M}$ belonging to the moving cluster have the same speed $\mathrm{v}_{S}$. The phase change resulting from the total travelled distance $d_{p}$ can be expressed as

$$
k_{0} d_{p}=\frac{2 \pi}{\lambda}\left(D_{l p}+D_{p k}\right)
$$


with

$$
\begin{aligned}
& D_{l p} \approx D_{p}^{T}-\left(M_{T}-2 l+1\right) \frac{\delta_{T}}{2} \cos \left(\alpha_{p}^{M}-\gamma_{T}\right) \\
& D_{p k} \approx D_{p}^{R}-\left(M_{R}-2 k+1\right) \frac{\delta_{R}}{2} \cos \left(\beta_{p}^{M}-\gamma_{R}\right)
\end{aligned}
$$

where $D_{p}^{T}$ and $D_{p}^{R}$ denote the distances from the scatterer $S_{p}^{M}$ to the transmitter and the receiver, respectively. After substituting (14)-(20) in (13) the channel gain due to the moving cluster can be written as

$$
g_{k l}^{\mathrm{M}}(t)=\sum_{p=1}^{P} \frac{a_{p}^{M} b_{p}^{M} c_{p}^{M}}{\sqrt{P}} e^{j\left(2 \pi\left(f_{p}^{T M}+f_{p}^{R M}-f_{p}^{T S}-f_{p}^{R S}\right) t+\theta_{p}\right)}
$$

where

$$
\begin{aligned}
a_{p}^{M} & =e^{j \pi \frac{\delta_{T}}{\lambda}\left(M_{T}-2 l+1\right) \cos \left(\alpha_{p}^{M}-\gamma_{T}\right)} \\
b_{p}^{M} & =e^{j \pi \frac{\delta_{R}}{\lambda}\left(M_{R}-2 k+1\right) \cos \left(\beta_{p}^{M}-\gamma_{R}\right)} \\
c_{p}^{M} & =e^{-j \frac{2 \pi}{\lambda}\left(D_{p}^{T}+D_{p}^{R}\right)} \\
f_{p}^{T M} & =f_{\max }^{T} \cos \left(\alpha_{p}^{M}-\phi^{T}\right) \\
f_{p}^{R M} & =f_{\max }^{R} \cos \left(\beta_{p}^{M}-\phi^{R}\right) \\
f_{p}^{T S} & =f_{\max }^{S} \cos \left(\alpha_{p}^{M}-\phi^{S}\right) \\
f_{p}^{R S} & =f_{\max }^{S} \cos \left(\beta_{p}^{M}-\phi^{S}\right) .
\end{aligned}
$$

It has to be noted that the $\operatorname{AoD} \alpha_{p}^{M}$ and the AoA $\beta_{p}^{M}$ are dependent since single-bounce scattering is assumed. The exact relationship between the AoD and the AoA can be found in (Chelli \& Pätzold, 2007). The envelope $\left|g_{k l}^{\mathrm{M}}(t)\right|$ follows a Rayleigh distribution due to the single-bounce scattering assumption.

\subsection{The Multiple-Cluster Channel Gain}

The channel gain $g_{k l}(t)$ has been derived assuming a scattering environment with two fixed clusters and one moving cluster. However, in real environment, one can find several buildings and several vehicles near to the mobile transmitter and receiver. Therefore, it is of interest to derive an expression for the channel gain in a multiple-cluster case $z_{k l}(t)$. The environment encompasses $\mathcal{C}^{T}\left(\mathcal{C}^{R}\right)$ fixed clusters around the transmitter (receiver) and $\mathcal{C}^{M}$ moving clusters. We added the subscripts $(\cdot)_{c^{T}},(\cdot)_{c^{R}}$, and $(\cdot)_{c^{M}}$ to all affected symbols to distinguish between the fixed clusters around the transmitter, the fixed clusters around receiver, and the moving clusters, respectively. The fixed cluster $c^{T}$ has a limited length $L_{c^{T}}$, it follows that the AoDs $\alpha_{m, c^{T}}^{T}$ are restricted to the interval $\left[\alpha_{\min , c^{T}}^{T}, \alpha_{\max , c^{T}}^{T}\right]$. Analogously, the AoDs $\beta_{n, c^{R}}^{R}, \alpha_{p, c^{M}}^{M}$, and $\beta_{p, c^{M}}^{M}$ are confined to the intervals $\left[\beta_{\min , c^{R}}^{R}, \beta_{\max , c^{R}}^{R}\right],\left[\alpha_{\min , c^{M}}^{M}, \alpha_{\max , c^{M}}^{M}\right]$, and $\left[\beta_{\min , c^{M}}^{M}, \beta_{\max , c^{M}}^{M}\right]$, respectively. Moreover, all the AoDs $\alpha_{m, c^{T}}^{T}(m=1,2, \ldots)$ have the same distribution and will be noted henceforth by $\alpha_{c^{T}}^{T}$. The same statement holds for the angles $\beta_{n, c^{R}}^{R}, \alpha_{p, c^{M}}^{M}$, and $\beta_{p, c^{M}}^{M}$ which will be denoted by $\beta_{c^{R}}^{R}, \alpha_{c^{M}}^{M}$, and $\beta_{c^{M}}^{M}$, respectively. 
In a multiple-cluster scenario the channel gain describing the link $A_{l}^{T}-A_{k}^{R}$ can be expressed as

$$
z_{k l}(t)=z_{k l}^{\mathrm{F}}(t)+z_{k l}^{\mathrm{M}}(t)
$$

where $z_{k l}^{\mathrm{F}}(t)$ is the received diffuse component due to double-scattering from all fixed clusters. The term $z_{k l}^{\mathrm{M}}(t)$ is the received diffuse component caused by single-scattering from all moving clusters. The channel gains $z_{k l}^{\mathrm{F}}(t)$ and $z_{k l}^{\mathrm{M}}(t)$ can be written as

$$
\begin{aligned}
z_{k l}^{\mathrm{F}}(t) & =\sum_{c^{T}, c^{R}=1}^{\mathcal{C}^{T}, \mathcal{C}^{R}} w_{c^{T}} w_{c^{R}} g_{k l, c^{T}, c^{R}}^{\mathrm{F}}(t) \\
z_{k l}^{\mathrm{M}}(t) & =\sum_{c^{M}=1}^{\mathcal{C}^{M}} w_{c^{M}} g_{k l, c^{M}}^{\mathrm{M}}(t)
\end{aligned}
$$

where $w_{c^{T}}, w_{c^{R}}$, and $w_{c^{M}}$ are positive constants representing the weighting factors of the clusters $c^{T}, c^{R}$, and $c^{M}$, respectively. We impose the boundary condition $\sum_{c^{T}, c^{T}=1}^{\mathcal{C}^{T}} \mathcal{C}^{R} w_{c^{T}}^{2} w_{c^{R}}^{2}+$ $\sum_{c^{M}=1}^{\mathcal{C}^{M}} w_{c^{M}}^{2}=1$, to normalize the mean power of $z_{k l}(t)$ to unity.

\section{Correlation Properties}

In this section, we derive analytical expressions for the correlation functions of the proposed MIMO V2V channel model, such as the 3D space-time CCF, the temporal ACF, and the 2D space CCF. The 3D space-time CCF $\rho_{k l, k^{\prime} l^{\prime}}\left(\delta_{T}, \delta_{R}, \tau\right)$ can be expressed as

$$
\begin{gathered}
\rho_{k l, k^{\prime} l^{\prime}}\left(\delta_{T}, \delta_{R}, \tau\right):=E\left\{z_{k l}^{*}(t) z_{k^{\prime} l^{\prime}}(t+\tau)\right\} \\
=\sum_{c^{T}, c^{R}=1}^{\mathcal{C}^{T}, \mathcal{C}^{R}} w_{c^{T}}^{2} w_{c^{R}}^{2} \rho_{k l, k^{\prime} l^{\prime}, c^{T}, c^{R}}^{\mathrm{F}}\left(\delta_{T}, \delta_{R}, \tau\right) \\
+\sum_{c^{M}=1}^{\mathcal{C}^{M}} w_{c^{M}}^{2} \rho_{k l, k^{\prime} l^{\prime}, c^{M}}^{\mathrm{M}}\left(\delta_{T}, \delta_{R}, \tau\right)
\end{gathered}
$$

where $(\cdot)^{*}$ denotes the complex conjugation and $E\{\cdot\}$ stands for the expectation operator. The term $\rho_{k l, k^{\prime} l^{\prime}, c^{T}, c^{R}}^{\mathrm{F}}\left(\delta_{T}, \delta_{R}, \tau\right)$ represents the 3D space-time CCF due to double-scattering from the clusters $c^{T}$ and $c^{R}$. This correlation function can be written as

$$
\begin{aligned}
\rho_{k l, k^{\prime} l^{\prime}, c^{T}, c^{R}}^{\mathrm{F}}\left(\delta_{T}, \delta_{R}, \tau\right) & :=E\left\{\left(g_{k l, c^{T}, c^{R}}^{\mathrm{F}}(t)\right)^{*} g_{k^{\prime} l^{\prime}, c^{T}, c^{R}}^{\mathrm{F}}(t+\tau)\right\} \\
& =\rho_{c^{T}}^{\mathrm{F}}\left(\delta_{T}, \tau\right) \cdot \rho_{c^{R}}^{\mathrm{F}}\left(\delta_{R}, \tau\right)
\end{aligned}
$$

where

$$
\rho_{c^{T}}^{\mathrm{F}}\left(\delta_{T}, \tau\right)=\int_{\alpha_{\min , c^{T}}^{T}}^{\alpha_{\max , c^{T}}^{T}} c_{l l^{\prime}}^{F}\left(\delta_{T}, \alpha_{c^{T}}^{T}\right) e^{j 2 \pi f^{T}\left(\alpha_{c^{T}}^{T}\right) \tau} p_{\alpha_{c^{T}}^{T}}\left(\alpha_{c^{T}}^{T}\right) d \alpha_{c^{T}}^{T}
$$


and

$$
\rho_{c^{R}}^{\mathrm{F}}\left(\delta_{R}, \tau\right)=\int_{\beta_{\min , c^{R}}^{R}}^{\beta_{\max , c^{R}}^{R}} d_{k k^{\prime}}^{F}\left(\delta_{R}, \beta_{c^{R}}^{R}\right) e^{j 2 \pi f^{R}\left(\beta_{c^{R}}^{R}\right) \tau} p_{\beta_{c^{R}}^{R}}\left(\beta_{c^{R}}^{R}\right) d \beta_{c^{R}}^{R}
$$

are the transmit and the receive correlation functions, respectively, and

$$
\begin{aligned}
c_{l l^{\prime}}^{F}\left(\delta_{T}, \alpha_{c^{T}}^{T}\right) & =e^{j 2 \pi \frac{\delta_{T}}{\lambda}\left(l-l^{\prime}\right) \cos \left(\alpha_{c^{T}}^{T}-\gamma_{T}\right)} \\
d_{k k^{\prime}}^{F}\left(\delta_{R}, \beta_{c^{R}}^{R}\right) & =e^{j 2 \pi \frac{\delta_{R}}{\lambda}\left(k-k^{\prime}\right) \cos \left(\beta_{c^{R}}^{R}-\gamma_{R}\right)} \\
f^{T}\left(\alpha_{c^{T}}^{T}\right) & =f_{\max }^{T} \cos \left(\alpha_{c^{T}}^{T}-\phi^{T}\right) \\
f^{R}\left(\beta_{c^{R}}^{R}\right) & =f_{\max }^{R} \cos \left(\beta_{c^{R}}^{R}-\phi^{R}\right) .
\end{aligned}
$$

The distributions of the AoD $\alpha_{c^{T}}^{T}$ and the AoA $\beta_{c^{R}}^{R}$ are denoted by $p_{\alpha_{c^{T}}^{T}}\left(\alpha_{c^{T}}^{T}\right)$ and $p_{\beta_{c^{R}}^{R}}\left(\beta_{c^{R}}^{R}\right)$, respectively.

In (32), the term $\rho_{k l, k^{\prime} l^{\prime}, c^{M}}^{\mathrm{M}}\left(\delta_{T}, \delta_{R}, \tau\right)$, which represents the 3D space-time CCF of the moving cluster $c^{M}$, can be expressed as

$$
\begin{gathered}
\rho_{k l, k^{\prime} l^{\prime}, c^{M}}^{\mathrm{M}}\left(\delta_{T}, \delta_{R}, \tau\right):=E\left\{\left(g_{k l, c^{M}}^{\mathrm{M}}(t)\right)^{*} g_{k^{\prime} l^{\prime}, c^{M}}^{\mathrm{M}}(t)(t+\tau)\right\} \\
=\int_{\alpha_{\min , c^{M}}^{M}}^{\alpha_{\max , c^{M}}^{M}} c_{l l^{\prime}}^{M}\left(\delta_{T}, \alpha_{c^{M}}^{M}\right) d_{k k^{\prime}}^{M}\left(\delta_{R}, g\left(\alpha_{c^{M}}^{M}\right)\right) \\
e^{j 2 \pi\left(f^{T M}\left(\alpha_{c^{M}}^{M}\right)+f^{R M}\left(g\left(\alpha_{c^{M}}^{M}\right)\right)-f^{T S}\left(\alpha_{c^{M}}^{M}\right)\right) \tau} \\
e^{-j 2 \pi f^{R S}\left(g\left(\alpha_{c^{M}}^{M}\right)\right) \tau} p_{\alpha_{c^{M}}^{M}}\left(\alpha_{c^{M}}^{M}\right) d \alpha_{c^{M}}^{M}
\end{gathered}
$$

where

$$
\begin{aligned}
c_{l l^{\prime}}^{M}\left(\delta_{T}, \alpha_{c^{M}}^{M}\right) & =e^{j 2 \pi \frac{\delta_{T}}{\lambda}\left(l-l^{\prime}\right) \cos \left(\alpha_{c^{M}}^{M}-\gamma_{T}\right)} \\
d_{k k^{\prime}}^{M}\left(\delta_{R}, g\left(\alpha_{c^{M}}^{M}\right)\right) & =e^{j 2 \pi \frac{\delta_{R}}{\lambda}\left(k-k^{\prime}\right) \cos \left(g\left(\alpha_{c^{M}}^{M}\right)-\gamma_{R}\right)} \\
f^{T M}\left(\alpha_{c^{M}}^{M}\right) & =f_{\max }^{T} \cos \left(\alpha_{c^{M}}^{M}-\phi^{T}\right) \\
f^{R M}\left(g\left(\alpha_{c^{M}}^{M}\right)\right) & =f_{\max }^{R} \cos \left(g\left(\alpha_{c^{M}}^{M}\right)-\phi^{R}\right) \\
f^{T S}\left(\alpha_{c^{M}}^{M}\right) & =f_{\max }^{S} \cos \left(\alpha_{c^{M}}^{M}-\phi^{S}\right) \\
f^{R S}\left(g\left(\alpha_{c^{M}}^{M}\right)\right) & =f_{\max }^{S} \cos \left(g\left(\alpha_{c^{M}}^{M}\right)-\phi^{S}\right) .
\end{aligned}
$$

The function $g(\cdot)$ in (40) expresses the exact relationship between the $\operatorname{AoD} \alpha_{c^{M}}^{M}$ and the AoA $\beta_{c^{M}}^{M}$. An expression for $g(\cdot)$ can be found in (Chelli \& Pätzold, 2007). 
The temporal ACF $r_{z_{k l}}(\tau)$ of the channel gain $z_{k l}(t)$ is defined as $r_{z_{k l}}(\tau):=E\left\{z_{k l}^{*}(t) z_{k l}(t+\tau)\right\}$ (Papoulis \& Pillai, 2002). The temporal ACF $r_{z_{k l}}(\tau)$ can be deduced from the 3D space-time CCF $\rho_{k l, k^{\prime} l^{\prime}}\left(\delta_{T}, \delta_{R}, \tau\right)$ by setting the antenna element spacings $\delta_{T}$ and $\delta_{R}$ to zero, i.e.,

$$
\begin{aligned}
r_{z_{k l}}(\tau)= & \rho_{k l, k^{\prime} l^{\prime}}(0,0, \tau) \\
= & \sum_{c^{T}, c^{R}=1}^{\mathcal{C}^{T}, \mathcal{C}^{R}} w_{c^{T}}^{2} w_{c^{R}}^{2} \rho_{k l, k^{\prime} l^{\prime}, c^{T}, c^{R}}^{\mathrm{F}}(0,0, \tau) \\
& +\sum_{c^{M}=1}^{\mathcal{C}^{M}} w_{c^{M}}^{2} \rho_{k l, k^{\prime} l^{\prime}, c^{M}}^{\mathrm{M}}(0,0, \tau) .
\end{aligned}
$$

The 2D space CCF $\rho_{k l, k^{\prime} l^{\prime}}\left(\delta_{T}, \delta_{R}\right)$ is defined as $\rho_{k l, k^{\prime} l^{\prime}}\left(\delta_{T}, \delta_{R}\right):=E\left\{z_{k l}^{*}(t) z_{k^{\prime} l^{\prime}}(t)\right\}$. Alternatively, the $2 \mathrm{D}$ space CCF $\rho_{k l, k^{\prime} l^{\prime}}\left(\delta_{T}, \delta_{R}\right)$ can be derived from the 3D space-time CCF $\rho_{k l, k^{\prime} l^{\prime}}\left(\delta_{T}, \delta_{R}, \tau\right)$ by setting $\tau$ to zero, i.e.,

$$
\begin{aligned}
\rho_{k l, k^{\prime} l^{\prime}}\left(\delta_{T}, \delta_{R}\right)= & \rho_{k l, k^{\prime} l^{\prime}}\left(\delta_{T}, \delta_{R}, 0\right) \\
= & \sum_{c^{T}, c^{R}=1}^{\mathcal{C}^{T}, \mathcal{C}^{R}} w_{\mathcal{c}^{T}}^{2} w_{c^{R}}^{2} \rho_{k l, k^{\prime} l^{\prime}, c^{T}, c^{R}}^{\mathrm{F}}\left(\delta_{T}, \delta_{R}, 0\right) \\
& +\sum_{c^{M}=1}^{\mathcal{C}^{M}} w_{c^{M}}^{2} \rho_{k l, k^{\prime} l^{\prime}, c^{M}}^{\mathrm{M}}\left(\delta_{T}, \delta_{R}, 0\right) .
\end{aligned}
$$

\section{Numerical Results}

In this section, we confirm the validity of the analytical expressions presented in the previous section by simulations making use of the sum-of-cisoids method. The simulation models for moving and fixed scatterers are designed using the modified method of equal area (MMEA) proposed in (Gutiérrez \& Pätzold, 2007). In order to model all fixed clusters, 50 cisoids are used for the simulation model. The same number of cisoids is used to model all moving clusters. The propagation environment contains six moving clusters: three clusters are located on the right side of the transmitter and the receiver, while the remaining clusters lie on the left side. Each moving cluster has a length of $5 \mathrm{~m}$ and is separated by a distance of $45 \mathrm{~m}$ from its neighbour clusters. The distance between the transmitter and the moving scatterers located on the left and the right side is set to $3 \mathrm{~m}$. For the fixed clusters, we consider a propagation environment encompassing three clusters on each side of the transmitter. Each cluster has a length of $2 \mathrm{~m}$ and is separated by a distance of $34 \mathrm{~m}$ from its neighbour clusters. The same number of fixed clusters is considered around the receiver. The distance between the transmitter and the fixed scatterers on the left and right side is set to $300 \mathrm{~m}$. The distance between the transmitter and the receiver is equal to $100 \mathrm{~m}$. The transmitter and the receiver have a speed of $50 \mathrm{~km} / \mathrm{h}$ and equal angles of motion $\phi^{T}=\phi^{R}=0$. The transmitter and the receiver antenna tilts $\gamma_{T}$ and $\gamma_{R}$ are set to $\pi / 2$. We consider the case of non-isotropic scattering conditions. The AoDs $\alpha_{c^{T}}^{T}$ and $\alpha_{c^{M}}^{M}$ are uniformly distributed over the intervals $\left[\alpha_{\min , c^{T}}^{T}, \alpha_{\max , c^{T}}^{T}\right]$ and $\left[\alpha_{\min , c^{M}}^{M}, \alpha_{\max , c^{M}}^{M}\right]$, respectively. The uniform distribution is also assumed for the AoAs $\beta_{c^{R}}^{R}$ over the interval $\left[\beta_{\min , c^{R}}^{R}, \beta_{\max , c^{R}}^{R}\right]$.

We present some illustrative examples for the temporal ACF $r_{z_{k l}}^{\mathrm{M}}(\tau)$ of the moving clusters in Fig. 3. We study the influence of the speed of the vehicles in the vicinity of the transmitter and 
the receiver on the channel behaviour. The term $v_{S}$ in Fig. 3 denotes the speed of the vehicles on the left and right side. From Fig. 3, we can notice that as the speed $v_{S}$ decreases, the coherence time of the channel increases. It is well known that the coherence time indicates whether we are facing a fast or a slow fading. As the speed of the vehicles relatively to the transmitter and the receiver decreases the channel changes more slowly. A good fitting between the simulation results and the theoretical results can be observed in Fig. 3. In Fig. 4, we show the

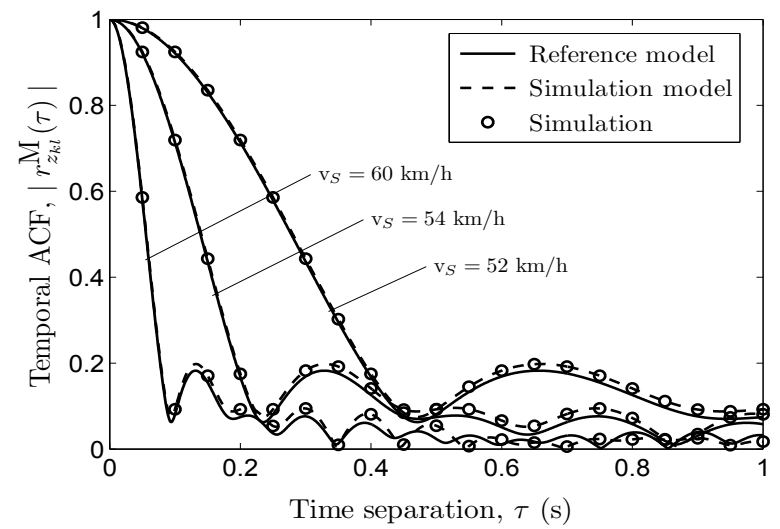

Fig. 3. The absolute value of the temporal ACF $r_{z_{k l}}^{\mathrm{M}}(\tau)$ associated with moving scatterers for various values of the velocity $\mathrm{v}_{S}$ of the surrounding vehicles.

numerical results obtained for the $2 \mathrm{D}$ space CCF $\rho_{11,22}^{\mathrm{M}}\left(\delta_{T}, \delta_{R}\right)$ caused by moving scatterers. It could be seen from Fig. 4 that the cross-correlation function decreases as we increase the antenna spacings $\delta_{T}$ and $\delta_{R}$. However, the decay of the $2 \mathrm{D}$ space CCF $\rho_{11,22}^{\mathrm{M}}\left(\delta_{T}, \delta_{R}\right)$ is faster along the $\delta_{R}$ direction. Hence, a small antenna spacing at the receiver side guarantees a diversity gain, but at the receiver side, we need a larger spacing to get non-correlated channels. In

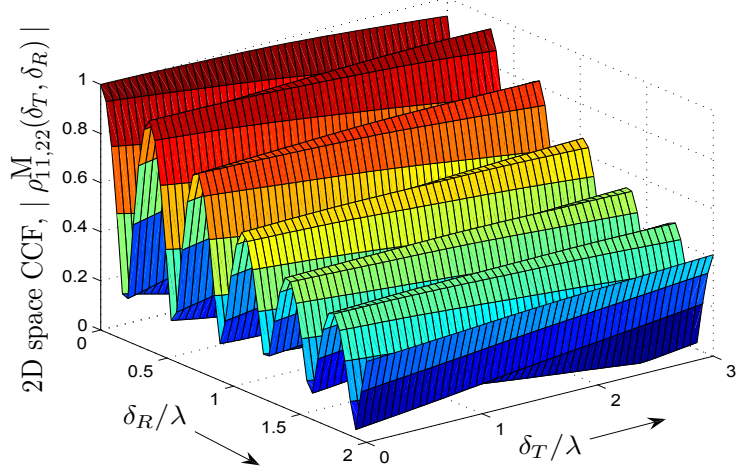

Fig. 4. The absolute value of the $2 \mathrm{D}$ space $\mathrm{CCF} \rho_{11,22}^{\mathrm{M}}\left(\delta_{T}, \delta_{R}\right)$ of the reference model caused by moving scatterers. 
Fig. 5, we illustrate the numerical results for the transmit correlation function $\rho_{T}^{\mathrm{F}}\left(\delta_{T}, \tau\right)$ of the reference model resulting from fixed scatterers. The obtained results are confirmed by simulation in Fig. 6. Similar results have been found for the receive correlation function $\rho_{R}^{\mathrm{F}}\left(\delta_{R}, \tau\right)$ associated with fixed scatterers since the same setting for the scatterers is considered around the transmitter and the receiver. Limited space prevents us from including these results.

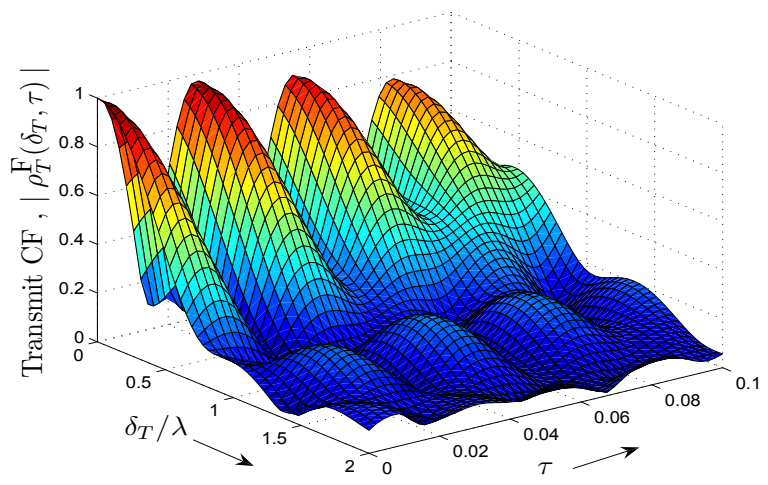

Fig. 5. The transmit correlation function $\rho_{T}^{\mathrm{F}}\left(\delta_{T}, \tau\right)$ of the reference model due to fixed scatterers.

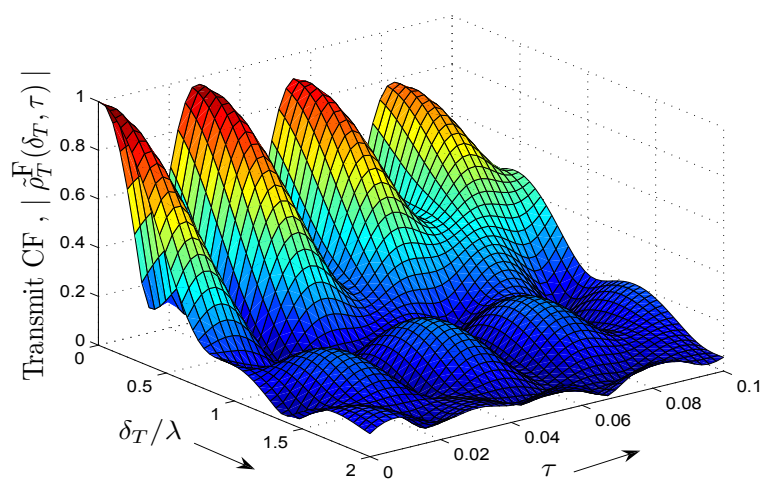

Fig. 6. The transmit correlation function $\tilde{\rho}_{T}^{\mathrm{F}}\left(\delta_{T}, \tau\right)$ of the simulation model related to fixed scatterers.

\section{Conclusion}

In this chapter, we have presented a narrowband MIMO V2V channel model, where both the impact of fixed and moving scatterers were taken into account. Double-bounce scattering is assumed for the fixed scatterers, while single-bounce scattering is considered for the moving 
scatterers. For reasons of brevity, we have restricted our investigations to non-line-of-sight situations. A reference model has been derived starting from the geometrical street model. The statistical properties of the proposed channel model have been studied. We have provided analytical expressions for the 3D space-time CCF, the temporal ACF, and the 2D space CCF. Supported by our analysis, we are convinced that the effect of moving scatterers on the statistics of V2V MIMO channels cannot be neglected. The investigation of the impact of moving scatterers have revealed that as the speed of the vehicles in the vicinity of the transmitter and the receiver decreases, the channel coherence time increases. The proposed channel model is suitable for a highway environment under congestion conditions. In such conditions, the low relative speed of the vehicles in the vicinity of the transmitter and the receiver allows us to consider that the AoD and the AoA seen from moving clusters are non-time-variant during a sufficiently large period of time. Actually, if the AoD and the AoA are time-variant, the channel model becomes non-stationary. The latter aspect will be investigated in future work.

\section{References}

802.11p (2006). Wireless access in vehicular environment (WAVE) in standard 802.11 information technology telecommunications and information exchange between systems, local and metropolitan area networks, specific requirements, part 11: Wireless lan medium access control (MAC) and physical layer (PHY) specifications, Technical Report p/D1.0, IEEE 802.11.

ASTM (2003). Standard specification for telecommunications and information exchange between roadside and vehicle systems - 5 ghz band dedicated short range communications (DSRC) medium access control (MAC) and physical layer (PHY) specifications. ASTM E2213-03.

Chelli, A. \& Pätzold, M. (2007). A MIMO mobile-to-mobile channel model derived from a geometric street scattering model, Proc. 4th IEEE International Symposium on Wireless Communication Systems, ISWCS'07, Trondheim, Norway, pp. 792-797.

Chelli, A. \& Pätzold, M. (2008). A wideband multiple-cluster MIMO mobile-to-mobile channel model based on the geometrical street model, Proc. 19th IEEE Int. Symp. on Personal, Indoor and Mobile Radio Communications, IEEE PIMRC 2008, Cannes, France.

Gutiérrez, C. A. \& Pätzold, M. (2007). Sum-of-sinusoids-based simulation of flat fading wireless propagation channels under non-isotropic scattering conditions, Proc. 50th IEEE Global Telecommunications Conference, GLOBECOM 2007, Washington, DC, USA, pp. 3842-3846.

Millott, L. (1994). The impact of vehicular scattering on mobile communications, Proc. 44th IEEE Veh. Technol. Conf., VTC'1994, Stockholm, Sweden, pp. 1733-1736.

Molisch, A., Kuchar, A., Laurila, J., Hugl, K. \& Bonek, E. (1999). Efficient implementation of a geometry-based directional model for mobile radio channels, Proc. 50th IEEE Veh. Technol. Conf., VTC'1999-Fall, Amsterdam, Netherlands, pp. 1449-1453.

Papoulis, A. \& Pillai, S. U. (2002). Probability, Random Variables and Stochastic Processes, McGraw-Hill, New York.

Patel, C. S., Stüber, G. L. \& Pratt, T. G. (2003). Simulation of Rayleigh faded mobile-to-mobile communication channels, Proc. 58th IEEE Veh. Technol. Conf., VTC'03, Orlando, FL, USA, pp. 163-167.

Pätzold, M., Hogstad, B. O., Youssef, N. \& Kim, D. (2005). A MIMO mobile-to-mobile channel model: Part I - The reference model, Proc. 16th IEEE Int. Symp. on Personal, Indoor and Mobile Radio Communications, PIMRC 2005, Berlin, Germany, pp. 573-578. 
Salo, J., El-Sallabi, H. \& Vainikainen, P. (2006). Impact of double-Rayleigh fading on system performance, Proc. 1st IEEE Int. Symp. on Wireless Pervasive Computing, ISWPC 2006, Phuket, Thailand.

WAVE (2005). Wireless access in vehicular environments (WAVE) channel coordination. IEEE P1609.4/D05.

Zajić, A. G., Stüber, G. L., Pratt, T. G. \& Nguyen, S. (2008). Statistical modeling and experimental verification of wideband MIMO mobile-to-mobile channels in highway environments, Proc. 44th IEEE Veh. Technol. Conf., VTC'1994, Cannes, France. 


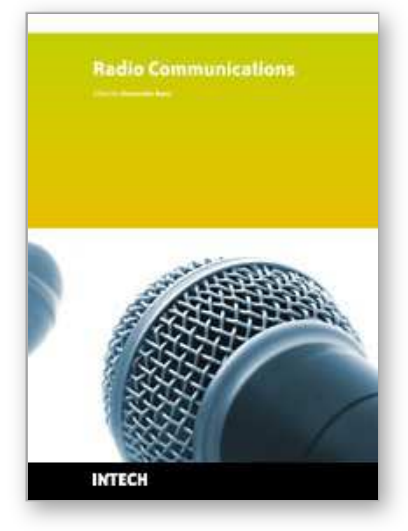

\author{
Radio Communications \\ Edited by Alessandro Bazzi
}

ISBN 978-953-307-091-9

Hard cover, 712 pages

Publisher InTech

Published online 01, April, 2010

Published in print edition April, 2010

In the last decades the restless evolution of information and communication technologies (ICT) brought to a deep transformation of our habits. The growth of the Internet and the advances in hardware and software implementations modified our way to communicate and to share information. In this book, an overview of the major issues faced today by researchers in the field of radio communications is given through 35 high quality chapters written by specialists working in universities and research centers all over the world. Various aspects will be deeply discussed: channel modeling, beamforming, multiple antennas, cooperative networks, opportunistic scheduling, advanced admission control, handover management, systems performance assessment, routing issues in mobility conditions, localization, web security. Advanced techniques for the radio resource management will be discussed both in single and multiple radio technologies; either in infrastructure, mesh or ad hoc networks.

\title{
How to reference
}

In order to correctly reference this scholarly work, feel free to copy and paste the following:

Ali Chelli and Matthias Patzold (2010). The Impact of Fixed and Moving Scatterers on the Statistics of MIMO Vehicle-to-Vehicle Channels, Radio Communications, Alessandro Bazzi (Ed.), ISBN: 978-953-307-091-9, InTech, Available from: http://www.intechopen.com/books/radio-communications/the-impact-of-fixed-andmoving-scatterers-on-the-statistics-of-mimo-vehicle-to-vehicle-channels

\section{INTECH}

open science | open minds

\author{
InTech Europe \\ University Campus STeP Ri \\ Slavka Krautzeka 83/A \\ 51000 Rijeka, Croatia \\ Phone: +385 (51) 770447 \\ Fax: +385 (51) 686166 \\ www.intechopen.com
}

\author{
InTech China \\ Unit 405, Office Block, Hotel Equatorial Shanghai \\ No.65, Yan An Road (West), Shanghai, 200040, China \\ 中国上海市延安西路65号上海国际贵都大饭店办公楼 405 单元 \\ Phone: +86-21-62489820 \\ Fax: $+86-21-62489821$
}


(C) 2010 The Author(s). Licensee IntechOpen. This chapter is distributed under the terms of the Creative Commons Attribution-NonCommercialShareAlike-3.0 License, which permits use, distribution and reproduction for non-commercial purposes, provided the original is properly cited and derivative works building on this content are distributed under the same license. 